\title{
Innovative Classroom Practices and the Teacher of the Future
}

\author{
Nancy Law \\ University of Hong Kong, Pokfulam Road, Hong Kong, nlaw@hkusua.hku.hk
}

\begin{abstract}
The widespread support for the use of ICT to support teaching and learning across the curriculum has been on the grounds that there is a need to equip the younger generation with a new set of competencies required for life in the $21^{\text {st }}$ century. There is also a heightening realization that this will also involve the development of new pedagogies alongside the use of ICT. It is in this context that the Second International Information Technology in Education Study introduced the concept of emerging pedagogical paradigm. The second phase of this study is an international comparative case study of 'Innovative Pedagogical Practices Using Technology' (SITES M2). Altogether 28 countries from around the world participated in this study, and a total of 174 case studies have been conducted and reported. Each of the innovative cases is by definition considered to be a relatively rare and valuable pedagogical development in its own national context and provides a lens for us to have a preview of the classrooms of the future. This paper explores the future roles of the teacher and the consequent demands on teacher professional development provisions based on an analysis of the variety of roles that teachers played in a sample of case studies collected in this study.
\end{abstract}

\section{INTRODUCTION}

This paper explores the future roles of the teacher and the consequent demands on the provision of teacher professional development based on a study of the variety of roles that teachers played in a sample of case studies collected in an international comparative study of innovative classroom practices using technology.

A main justification for the widespread introduction of ICT to support teaching and learning across the curriculum has been the development of a new set of competencies to prepare the younger generation for life in the $21^{\text {st }}$

The original version of this chapter was revised: The copyright line was incorrect. This has been corrected. The Erratum to this chapter is available at DOI: 10.1007/978-0-387-35701-0_35 
century. It is undeniable that there is a growing tendency for education policies on ICT in the curriculum to be linked to or made in the context of a wider educational renewal/reform at a national level (e.g. Ireland, 1999; Korean Ministry of Education, 2000; PCAST, 1997; Singapore Ministry of Education, 1997). It is also in this context that the Second International Information Technology in Education Study introduced the concept of an emerging pedagogical paradigm for the study (Pelgrum \& Anderson, 1999) and conceptualized the second phase of this study: an international comparative case study of 'Innovative Pedagogical Practices Using Technology' (hereafter referred to as SITES M2). Altogether 28 countries from around the world participated in this study, contributing to a total of 174 case studies being conducted in 2000 and 2001 (s e e http://www.sitesm2.org//SITES_Research_Projects/SITESM2/prospectus.html).

As each of the innovative cases is by definition considered to be relatively rare and valuable pedagogical developments in its own national context, the cases provide a lens for us to have a preview of the classrooms of the future. In analyzing the case studies, the Hong Kong research team wanted to address the issue of innovativeness: in what sense can we say that these classrooms are futuristic? What can be learnt from these cases that can help us to transform our classrooms to better prepare students for the challenges of the $21^{\text {st }}$ century? This paper examines in particular the roles played by teachers in the innovative case studies analyzed to provide a better understanding of the changing roles demanded of the teacher of the future and the consequent demands on teacher professional development provisions.

\section{INNOVATIVE PEDAGOGICAL PRACTICES: A FRAMEWORK FOR ANALYSIS}

In order to arrive at a framework for analyzing innovations, the research team found it necessary to examine innovation from the perspective of change and to examine the key dimensions of change that may contribute to classroom practices using technology to be considered as innovative.

Plomp, ten Brummelhuis and Rapmund (1996) discussed the concept of emergent pedagogical practices and the management of change. They introduced two important concepts: 'care for the old' and 'courage for the new'. The basic idea is that in implementing ICT in educational settings, there needs to be a clear focus on bringing about new learning outcomes and new modes of learning, and not just on the adoption of new technologies. However, it is also recognized that schools and schooling cannot be changed overnight and that the process of innovation has to be a gradual one. Thus it 
was recognized that in the early stages of the implementation process, ICT would most likely be used for the delivery of old classroom practices for the achievement of long existent goals. Plomp et al. (1996) further argued for this kind of implementation to be supported so that the people involved in the process of change can ease into the change. This kind of practice was labeled as 'care for the old'. On the other hand, the same work also pointed out very clearly that it is those practices which bring about new learning goals and new modes of learning that are the most important and any educational institution needs to have courage and determination to bring about such new practices, as these will be the practices that will define and shape the future of schooling.

Adopting this framework to examine the case studies of innovative practices collected in SITES M2, we identify 6 dimensions (or aspects) of classroom practices that might have changed as part of the innovation:

Intended learning objectives of the classroom practice

Pedagogical role(s) of the teacher

Role(s) of the learner

Nature and sophistication of the ICT used

Connectedness of the classroom

Learning outcomes exhibited by the learner

These 6 dimensions of change are key elements in any curriculum implementation involving ICT use. When the features of each case study were examined along each of these 6 dimensions, it became apparent that there was large diversity across these cases. Taking the 'traditional' classroom to be typically one that is isolated, knowledge-focused, teachercentered, does not use ICT and only assesses students on cognitive learning outcomes, Table 1 lists the features of the practices studied on a 'scale of innovativeness' on the basis of the 'magnitude of change' that has taken place along each of the 6 dimensions of analysis. It is apparent from Table 1 that some of the features observed were very similar to traditional practices, while others may have quite innovative features that are rarely found in current classrooms.

\section{TEACHERS' ROLES AS A FOCAL DIMENSION AND TEACHERS' CAPABILITIES}

While all the 6 dimensions identified are necessarily present in any classroom practice, they are not entirely independent of each other. In fact, the role played by the teacher is arguably the most important dimension since it is the teacher who orchestrates and exerts the greatest influence on the other 5 dimensions. Obviously, it is important to recognize that the 


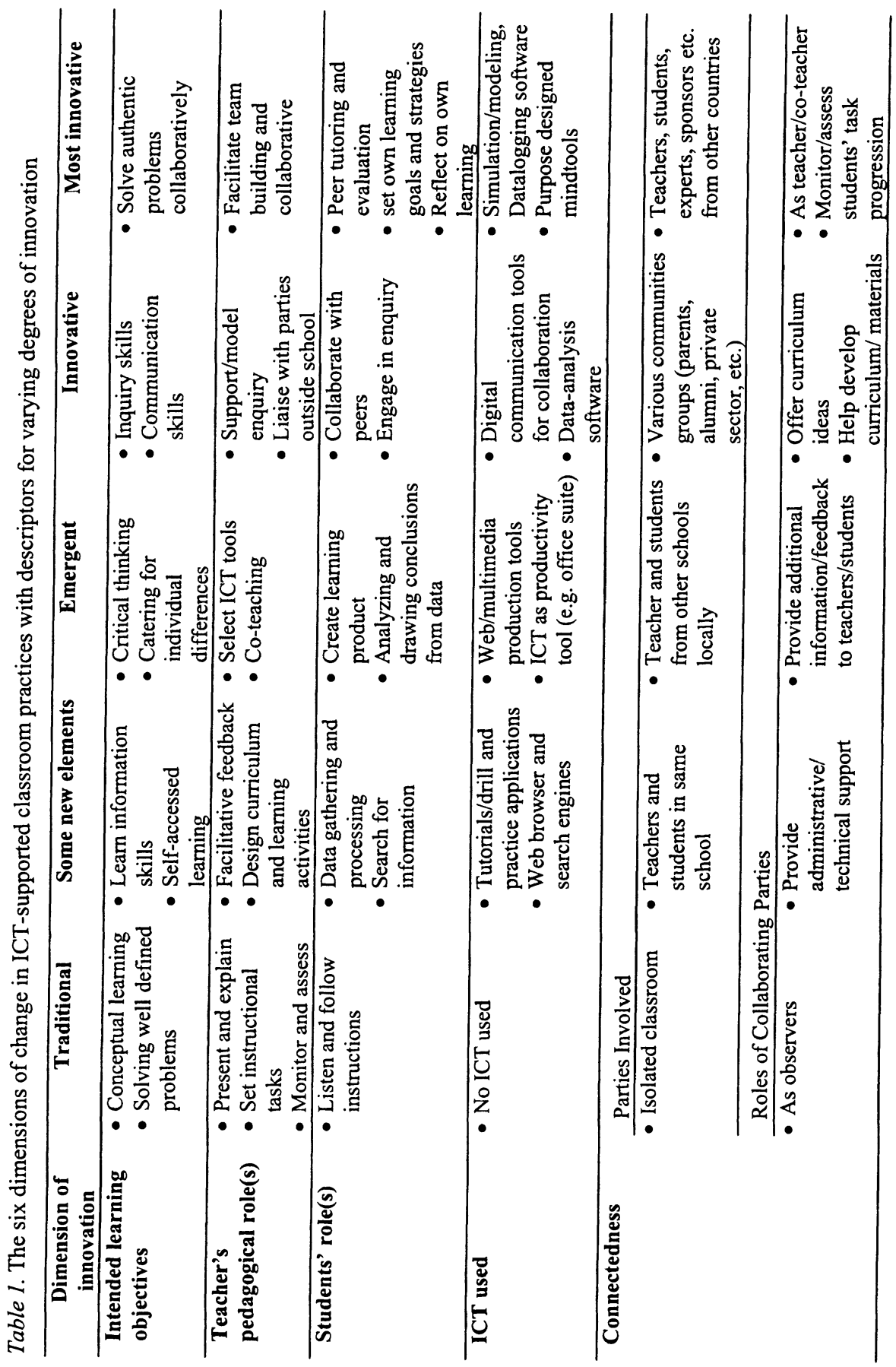


teacher operates within the context of the school and is constrained by the vision and goals as well as the resources and priorities of the institution involved, but such factors lie outside the scope of this paper.

In examining the innovative classroom practices collected in SITES M2, the Hong Kong research team observed great diversity across different case studies in the profile of features along the 6 dimensions. It was not common to find classroom practices that possessed highly innovative features along all the 6 dimensions. On the other hand, many of the case studies were highly innovative along one or a few of the 6 dimensions. Another important observation was that even though in some case studies the teachers' pedagogical roles (which encompass the most important aspects of their roles in relation to the pedagogical approach adopted, but not the entire set of roles played by the teachers) remained rather traditional, the innovations that occurred along the other dimensions in those cases also created new demands on the teacher. These new demands then required the teachers to develop new capabilities and competences in the implementation process. Therefore, irrespective of whether there were substantial changes in the pedagogical roles played by the teacher, the teacher had to innovate at a professional level to make the classroom innovation a reality. In the following sections, 3 case studies will be examined to illustrate the diversities in the roles played by the teacher and the range of new capabilities demanded of the teacher in those cases.

\section{NURTURING LEARNING COMMUNITIES}

One of the innovative practices selected for the Hong Kong SITES M2 study was a Cyber Art Project in a primary school. The innovation involved the introduction of wireless laptop computers fitted with a pressure pad and associated painting and drawing software for the learning of Art in all classes from grades 4 to 6 in the school. The target was for up to $25 \%$ of the Art lessons in these classes to be conducted using this new medium. The aim of the innovation was to promote students' creativity, interest and development of skills through the greater flexibility in the formats and contexts available for art creation offered by the new medium. The pupils were able to experiment with different simulated forms of painting such as graphite sketches, Chinese painting, watercolors and oil painting on the computer. Further, they were able to easily practice drawing outside the Art room: at the school playground, at the Peak, at the Art Gallery, as well as take part in a drawing excursion to the Great Wall in Beijing. The wireless network also allowed pupils to share their artwork with other classmates and the teachers also created opportunities for pupils to discuss and critique each 
other's work. In addition, the school organized an Art excursion to Beijing that included sharing activities in Art with a Beijing primary school.

This project was led by the 3 Art teachers who taught Art in the 3 target grades (4 to 6), including the Art Panel chairperson of the school who initiated the whole project. Besides these 3 teachers, an additional 14 teachers were also peripherally involved in supporting the Art excursions and took part in some associated professional development activities so as to prepare themselves for playing a more active role in using computers for the teaching of Art when its use became extended to the lower grades in future. These 17 teachers worked closely together to prepare the curriculum materials and the learning activities. They would visit each other's classes to conduct evaluation. Another prominent feature of this practice was that the teachers had to undertake a lot of liaison work with external parties in order to organize the excursions, including parents (who went to Beijing with the students), the Curator of the Art Gallery and the principal and teachers of the partner school in Beijing that hosted their visit.

The pedagogical role played by the teachers in this practice is not considered to be particularly innovative. The practice was still relatively teacher-centered in that the teacher determined the nature and scope of the activity and the pupils largely followed the instructions. The learning outcomes targeted were essentially the same as what one normally finds stated in the Art curriculum. What is outstanding in this practice, however, was the conception of and commitment to extending the pupils' opportunities to learn by extending the learning contexts to beyond the traditional classroom. Here the technology used was not commonly found in Hong Kong schools and the teachers had only marginal knowledge of the technology before the funding for the project was approved. The technology used was specifically designed for creating different Art forms through the digital medium. The teachers had to learn and master this new technology and to creatively design appropriate curriculum activities for the classes concerned using their curriculum development and pedagogical expertise.

Another prominent feature in this practice is the courage that the teachers took in opening up their classroom doors. In so doing, the teachers did not only have to take their pupils out of the classroom, but also greatly increased the range of participants involved in the teaching and learning process. Where traditionally a classroom includes a teacher and a class of pupils, the 'classroom' in this practice extended beyond the school premises and included many more participants such as teachers in the same school other than the class Art teacher, parents, teachers and pupils from another school, as well as people outside the education community such as museum curators. The 'openness' thus created provides a greater opportunity not only for the pupils to learn, but also for the school and the teachers to learn and improve 
as an organization. This new 'openness' also demanded that teachers be capable of identifying and soliciting collaborative partners to support the planned learning and teaching activities.

A third major challenge that the three core teachers took in their stride was to nurture the establishment of a community of learners within the Art panel team during the innovation process. They worked closely together to tackle the technical and professional difficulties encountered and in so doing created a community of professionals who were capable of identifying their own goals for organizational development as well as individual professional advancement.

In sum, the teachers in this innovative practice have developed remarkable capabilities in helping the school to develop into a learning organization and in bringing extensive community involvement into the learning and teaching process.

\section{DEVELOPING ACADEMIC PROFESSIONAL EXPERTISE}

The second case study to be discussed here, Computer Assisted Scientific Investigations, was rather different from the previous one in nature. This innovation involved 3 senior science teachers teaching the three science subjects biology, chemistry and physics respectively in grades 10-13 in a secondary school in Hong Kong. Here, the teachers were not satisfied with the rather didactic and prescriptive way that experimental work in science was generally organized in Hong Kong schools. They very much wished to introduce scientific investigations into the curriculum so that students could generate the problem to be investigated as well as formulate the method of enquiry, in addition to conducting the experiment and interpreting the results to arrive at a conclusion. They believed that the introduction of datalogging in science laboratories would greatly extend the scope of experimentation accessible to the students and would thus enable scientific investigations to be introduced into the curriculum.

Students were recruited voluntarily from grades 10 and 12 into this pilot project and formed into 3 teams, one for each science subject. Each team of students was to further subdivide into 10 groups to develop 10 investigations. First of all, each group had to identify a problem that the members consider to be interesting and/or important to investigate. They then had to work out a method for the investigation, conduct the experiment, and analyze the data collected to determine whether the results so obtained were able to address the problem they started with. In the event that they encountered problems, they might modify or improve their design until they 
were able to find an answer to their problem. Each group had to produce, as a final product, a laboratory guide for the investigation so that other students could follow the instructions and conduct the experiment accordingly.

While there was some collaboration among the three science teachers in terms of setting up a common structural framework for the pilot project, and in familiarizing themselves with the use of the datalogging equipment, the three teachers worked relatively independently with their own team of students. The greatest challenge was a pedagogical one, changing the role of the teacher from an instructor and evaluator to that of a learning guide to the students. The teachers did not know for sure the answers to the problems to be investigated, as these were authentic ones generated by the students. They modeled the investigation process through sharing and supporting it. However, they did not take over the investigation but rather helped students to clarify their ideas by posing questions and occasionally suggesting possible courses of attack. They also provided resources and technical skills training when required by students.

This pedagogical role change on the part of the teachers brings with it socio-emotional challenges to the teacher as they will no longer be the authority on the subject matter to be taught and will become more like a colearner alongside the students. In taking up new pedagogical roles, these teachers were also helping students to assume new roles as learners. Students were no longer passive consumers of packaged instruction but learn to become autonomous knowledge workers in a team by contributing productively to the creation of a solution that addresses an authentic problem.

Another challenge that these teachers overcame was academic and professional in nature. The teachers had not used dataloggers at all and only knew about them vaguely through reading in professional journals. They were not particularly technology savvy individuals but took on the professional commitment to keep abreast of the latest developments in their discipline area for the benefit of their students. In so doing, they learnt about the use of rather sophisticated technology that extended the scope of investigations and the extent of knowledge of the discipline areas that they taught in. These teachers, through their own professional conduct and activities, demonstrated clearly that they were practicing lifelong learners.

\section{ROLE DIFFERENTIATION IN CYBER INSTITUTIONS}

The last case study to be discussed here is one drawn from Australia. This involves a Virtual Schooling Service in a statewide project. Eighteen 
teachers were involved in planning the virtual classes and online learning materials in six subject areas for 320 secondary students in 49 schools throughout the state. These 49 schools had difficulty in offering the full range of subjects because of the small student numbers involved. This project was an attempt to extend students' educational opportunities through the use of flexible delivery strategies and a range of synchronous and asynchronous learning technologies. Students were grouped across schools to make up viable virtual classes. Two groups of teachers were involved in this practice: 18 VSS (virtual schooling service) teachers employed for this statewide project and the school teachers from the 49 schools who acted as 'Study Coaches' for students in their own schools.

The 18 VSS teachers were responsible for developing all the curriculum materials for both synchronous and asynchronous delivery. They also played many of the major roles normally assumed by classroom teachers for the students: conducting (online virtual) classes, providing instructions, setting assignments, communicating with students and providing feedback as well as assessing students' learning outcomes.

Students typically had two 'real time' virtual classes of approximately 40 minutes of synchronous lessons and three periods of offline, Study Room activities each week for each VSS subject enrolled. The virtual classes were presided over by a VSS teacher and students were able to interact with the VSS teacher and other students in the virtual class via 'real time' audio teleconferencing and shared computer graphics. During the virtual lessons, students had access to electronic resources, which could be received through emails from the VSS teacher or via some online subject resource repositories. Between lessons, students were able to interact with the remote VSS teacher and fellow students through a range of synchronous and asynchronous technologies, including email discussion lists and 'real-time' chat rooms.

In addition to the virtual classes, independent study time was organized in an online Study Room to engage students in activities and assignments. The Wide Area Network technology used in this project limited the students' access to the online Study Room to allocated asynchronous times of study, before and after school, and at lunch times using the school computers. The participating schools appointed Study Coaches from their own staff to provide assistance and guidance locally to students. Where students were less computer literate or less able to undertake independent learning, the local Study Coaches had to spend much of their time to provide technical support as well as subject-related assistance. The Study Coaches' roles also included supervision and guidance to students on management of their workloads and authentication of assessment items, as well as liaison with the VSS teachers on potential student problems. Student assignment tracking 
and ensuring assignment completion was a cooperative arrangement between the school-based Study Coach and the VSS teachers. Examinations were set by the VSS teachers and hard copies delivered to the schools' Study Coaches who then supervised the students in the completion of these and sent them back to the VSS teachers for marking.

In one sense, the key innovation involved in this activity was a technological one, using a range of multimedia and course development tools to present course materials, and various forms of synchronous and asynchronous communication tools to support teaching and learning interactions. The goals of the practice were very similar to the original curriculum goals for the subjects when offered in a face-to-face mode. In terms of pedagogical approaches, the VSS teachers were trying their best to replicate the best possible face-to-face approaches that could be used for the teaching of their subjects to the online situation. However, for effective learning to take place, this new mode of learning requires the learners to develop a greater sense of autonomy and self-direction as well as the skills and dispositions for lifelong learning. Furthermore, this activity involved explorations in employing various forms of technology to conduct different kinds of teaching and learning interactions, as well as exploration of ways of supplementing the cyber interactions with a local Study Coach who played roles that cyber interactions could not fully deliver. It is argued here that such explorations are important for generating innovations in learning technologies, in pedagogy, as well as in developing new types of education professionals leading to new ecologies and cultures in education communities.

First of all, the VSS teachers had to master new skills in a range of software (e.g. PowerPoint, NetMeeting) and to determine how to use this software most effectively for various teaching options. While materials and ideas they brought from their previous school-based teaching were useful, they also made adaptations as experience with the technology was gained. As fluency in the use of technology increased, they started to innovate new strategies for teaching and learning in the VSS context. Arguably, much of this courseware development role could be dissociated to a great extent from the actual teaching in virtual classes, leading to the establishment of a new type of education professional who specializes in the development of online course materials.

The physical separation of the VSS teachers from the students also forced them to reflect much more deeply on the strategies they employed and how they orchestrated different forms of communications with students for different purposes in order that they could deliberate more consciously and effectively in their communications (synchronous and asynchronous) with students. These VSS teachers were in fact developing new, specialized 
pedagogical competencies and capabilities and in the process evolved into a new breed of education professionals for cyber education institutions.

While the Study Coaches did not contribute to the design of the curriculum or to the teaching and assessment of the students, they played important supportive roles in coaching students to become autonomous learners and in liaising with the VSS teachers. Thus one can already observe a differentiation of the classroom teacher's roles into two components, one being played by the VSS teacher and the other played by the Study Coach. It may be possible that, as cyber education institutions grow and develop, Study Coaches will become a new type of education professional with their own unique skills, competencies and capabilities.

The process of differentiation and emergence of new education professionals as described above is an evolutionary process resulting from the process of innovation involving the adoption of new technologies to broaden the education opportunities of learners in remote areas. It is hypothesized here that such innovations will create pressure on the learning technologies to innovate as well, since the education professionals engaged in extensive use of technology will make demands on the technology to support the new pedagogies that they will in time innovate. The differentiation of teachers' roles into a number of different education professionals also makes it imperative that these professionals communicate and collaborate effectively, and it is expected that in time a new professional ecology may develop as a consequence of the emergence of cyber education institutions.

\section{INNOVATIONS AND TEACHERS' ROLES}

In conclusion, we have found analyzing the case studies of innovative pedagogical practices using technology selected from the SITES M2 study along the six dimensions, intended learning objectives, teacher's pedagogical roles, student's roles, nature and sophistication of the ICT used, the connectedness of the classroom and the learning outcomes exhibited by the learners to be very fruitful. The different case studies were found to exhibit particularly innovative features along some of the dimensions only, which may not include prominent innovations in the pedagogical role of the teacher. However, our study found that all the innovations were making new demands on the teacher, who had to be continuously engaged in lifelong learning as well as working collaboratively with other teachers in order to realize the innovative practices studied. The richness and success of these practices is not simply a result of the implementation of ICT in the teaching and learning process, but a consequence of the case selection criteria used in 
SITES M2. One important criterion for case selection was that the practice had to demonstrate evidence of significant changes in roles of teachers and students, the goals of the curriculum, assessment practices, and/or the educational materials or infrastructure. It is the author's view that it is through such kinds of pedagogical innovations that the teaching profession renews and recreates itself into a variety of education professionals who will lead schools into become learning organizations of the $21^{\text {st }}$ century.

\section{REFERENCES}

Ireland, I. S. (1999). Building a Capacity for Change: Lifelong Learning in the Information Society. Dublin: Stationery Office, Government of Ireland.

Korean Ministry of Education (2000). Adapting Education to the Information Age: A White Paper. Seoul: Korea Education \& Research Information Service.

PCAST (President's Committee of Advisors on Science and Technology Panel on Educational Technology) (1997). Report to the President on the Use of Technology to Strengthen K-12 Education in the United States.

Pelgrum, H. \& Anderson, R. (Ed.) (1999). ICT and the Emerging Paradigm for Life Long Learning. Amsterdam: IEA.

Plomp, T., ten Brummelhuis, A. \& Rapmund, R. (Eds.) (1996). Teaching and Learning for the Future. Den Haag: Committee on Multimedia in Teacher Training, Dutch Ministry of Education.

Singapore Ministry of Education (1997). IT Masterplan for Education. Singapore. 